This article has been accepted for publication in MNRAS (c) 2018 The Authors. Published by Oxford University Press on behalf of the Royal Astronomical Society. All rights reserved. 


\title{
The kinematic Sunyaev-Zel'dovich effect of the large-scale structure (II): the effect of modified gravity
}

\author{
M. Roncarelli ${ }^{\circledR},{ }^{1,2 \star}$ M. Baldi ${ }^{\oplus 1,2,3}$ and F. Villaescusa-Navarro ${ }^{4,5,6}$ \\ ${ }^{1}$ Dipartimento di Fisica e Astronomia, Università di Bologna, viale Berti Pichat 6/2, I-40127 Bologna, Italy \\ ${ }^{2}$ Istituto Nazionale di Astrofisica (INAF) - Osservatorio di Astrofisica e Scienza dello Spazio (OAS), via Gobetti 93/3, I-40127 Bologna, Italy \\ ${ }^{3}$ Istituto Nazionale di Fisica Nucleare (INFN) - Sezione di Bologna, viale Berti Pichat 6/2, I-40127 Bologna, Italy \\ ${ }^{4}$ Center for Computational Astrophysics, 160 Fifth Avenue, New York, NY 10010, USA \\ ${ }^{5}$ Istituto Nazionale di Astrofisica (INAF) - Osservatorio Astronomico di Trieste, via Tiepolo 11, I-34131 Trieste, Italy \\ ${ }^{6}$ Istituto Nazionale di Fisica Nucleare (INFN) - Sezione di Trieste, via Valerio 2, I-34127 Trieste, Italy
}

Accepted 2018 August 7. Received 2018 July 31; in original form 2018 May 29

\begin{abstract}
The key to understand the nature of dark energy lies in our ability to probe the distant Universe. In this framework, the recent detection of the kinematic Sunyaev-Zel'dovich (kSZ) effect signature in the cosmic microwave background obtained with the South Pole Telescope (SPT) is extremely useful since this observable is sensitive to the high-redshift diffuse plasma. We analyse a set of cosmological hydrodynamical simulation with four different realizations of a Hu \& Sawicki $f(R)$ gravity model, parametrized by the values of $\bar{f}_{\mathrm{R}, 0}=\left(0,-10^{-6},-10^{-5}\right.$, $-10^{-4}$ ), to compute the properties of the kSZ effect due to the ionized Universe and how they depend on $\bar{f}_{\mathrm{R}, 0}$ and on the redshift of reionization, $z_{\mathrm{re}}$. In the standard General Relativity limit $\left(\bar{f}_{\mathrm{R}, 0}=0\right)$ we obtain an amplitude of the $\mathrm{kSZ}$ power spectrum of $\mathcal{D}_{3000}^{\mathrm{kSZ}}=4.1 \mu \mathrm{K}^{2}\left(z_{\mathrm{re}}=8.8\right)$, close to the $+1 \sigma$ limit of the $\mathcal{D}_{3000}^{\mathrm{kSZ}}=(2.9 \pm 1.3) \mu \mathrm{K}^{2}$ measurement by SPT. This corresponds to an upper limit on the $\mathrm{kSZ}$ contribute from patchy reionization of $\mathcal{D}_{3000}^{\mathrm{kSZ} \text {,patchy }}<0.9 \mu \mathrm{K}^{2}(95$ per cent confidence level). Modified gravity boosts the kSZ signal by about 3,12 , and 50 per cent for $\bar{f}_{\mathrm{R}, 0}=\left(-10^{-6},-10^{-5},-10^{-4}\right)$, respectively, with almost no dependence on the angular scale. This means that with modified gravity the limits on patchy reionization shrink significantly: for $\bar{f}_{\mathrm{R}, 0}=-10^{-5}$ we obtain $\mathcal{D}_{3000}^{\mathrm{kSZ}, \text { patchy }}<0.4 \mu \mathrm{K}^{2}$. Finally, we provide an analytical formula for the scaling of the kSZ power spectrum with $z_{\text {re }}$ and $\bar{f}_{\mathrm{R}, 0}$ at different multipoles: at $\ell=3000$ we obtain $\mathcal{D}_{3000}^{\mathrm{kSZ}} \propto z_{\mathrm{re}}{ }^{0.24}\left(1+\sqrt{\left|\bar{f}_{\mathrm{R}, 0}\right|}\right)^{41}$.
\end{abstract}

Key words: methods: numerical - cosmic background radiation-dark ages, reionization, first stars - large-scale structure of Universe - cosmology: theory.

\section{INTRODUCTION}

The origin of the accelerated expansion of the Universe has become a longstanding problem in cosmology and in theoretical physics in general: we are about to mark the 20th anniversary of the first detection (by means of the dimming of distant supernovae (Riess et al. 1998; Schmidt et al. 1998; Perlmutter et al. 1999) of such pivotal discovery that shaped the development of a whole field of research, and that provided support and motivations for the design and funding of several challenging and costly observational enterprises to survey large portions of the sky, both from the ground (DES, LSST, HetDEX) and from space (Euclid, see Laureijs et al. 2011).
On the theoretical side, the past two decades have been characterized by widespread efforts to provide a more solid and natural framework for the accelerated expansion with respect to the highly fine-tuned (and yet most economic) option of the cosmological constant $\Lambda$ that characterizes the current standard cosmological model. Possible alternative scenarios have attempted to invoke the slow roll of a light scalar field (known as Quintessence Wetterich 1988; Ratra $\&$ Peebles 1988) and its possible couplings to the matter sector (see e.g. Wetterich 1995; Amendola 2000; Amendola, Baldi \& Wetterich 2008) as an explanation for the energy scale of the Dark Energy and for the relatively recent onset of the accelerated expansion, known as the fine-tuning and coincidence problems, respectively.

An alternative option amounts to considering possible deviations from the standard theory of gravity in the form of extensions to General Relativity (GR) that may result in an effective weakening of gravity at cosmological scales and late cosmic epochs. Such 
approach, generally known as Modified Gravity, has been extensively explored over the past years (see e.g. Amendola et al. 2013) allowing to identify and classify a wide range of geometric theories of gravity that deviate from standard GR still providing healthy equations of motion and sensible cosmological evolutions. As any deviation from the predicted behaviour of GR within the Solar System is very tightly constrained by local tests of gravity (see e.g. Bertotti, Iess \& Tortora 2003; Will 2006), all such theories must rely on some mechanism to recover standard GR within the local environment, which go under the general term of screening (see e.g. Vainshtein 1972; Damour \& Polyakov 1994; Khoury \& Weltman 2004; Nicolis, Rattazzi \& Trincherini 2009; Hinterbichler \& Khoury 2010). Unfortunately, when such constraining conditions are applied, most modified gravity theories still require a fine-tuned low-energy scale to reproduce the observed background expansion history, thereby failing to ease the problems of the cosmological constant. None the less, these scenarios still provide a theoretically consistent framework to test gravity on large scales and constrain possible deviations from the standard theory of GR. Besides the Solar System tests, the recent detection of the gravitational wave event GW170817 (Abbott et al. 2017) and of its electomagnetic counterpart has also severely constrained the landscape of possible modified gravity theories (see e.g. Baker et al. 2017; Creminelli \& Vernizzi 2017; María Ezquiaga \& Zumalacárregui 2017; Sakstein \& Jain 2017) by ruling out with a single observation all modified gravity models featuring a non-negligble difference of the propagation velocities of electromagntic and gravitational signals (see also Lombriser \& Taylor 2016; Lombriser \& Lima 2017). After this selection, only a bunch of modified gravity scenarios can be still considered as viable candidates for an extension of GR at cosmological scales (see e.g. fig. 2 in María Ezquiaga \& Zumalacárregui 2017).

The most widely studied example of such models still passing the GW170817 scrutiny is given by $f(R)$ gravity (Buchdahl 1970), where the standard Ricci curvature term $R$ in the Einstein-Hilbert Action is extended by an additional function $f(R)$ :

$S=\int \mathrm{d}^{4} x \sqrt{-g}\left(\frac{R+f(R)}{16 \pi G}+\mathcal{L}_{m}\right)$.

In equation (1) $G$ is Newton's gravitational constant, $g$ is the determinant of the metric tensor $g_{\mu \nu}$, and $\mathcal{L}_{m}$ is the Lagrangian density of all matter fields. The quantity $f_{R} \equiv \mathrm{d} f(R) / \mathrm{d} R$ represents a new scalar degree of freedom that propagates as the carrier of an additional force. In the weak-field and quasi-static limit, this scalar field obeys an independent dynamic equation ${ }^{1}$ (see Hu \& Sawicki 2007):

$\nabla^{2} f_{R}=\frac{1}{3}(\delta R-8 \pi G \delta \rho)$,

where $\delta R$ and $\delta \rho$ are the relative perturbations in the scalar curvature and matter density, respectively.

Different choices for the functional form of $f(R)$ in equation (1) may then lead to a large variety of effects on both the background expansion history of the Universe and the growth of its density perturbations, giving thus rise to possible characteristic observational signatures in the resulting large-scale structure (LSS) formation. Among the many ways to parametrize the variety of possible $f(R)$ forms, the most common approach (see Section 2 for a more detailed discussion) adopts the mean value of $f_{R}$ at the

${ }^{1}$ For the formulas presented in this section, we work in units where the speed of light is set to unity, $c=1$. present epoch, $\bar{f}_{\mathrm{R}, 0}$, as the key parameter to describe deviations from standard GR, and uses it as a reference to define observational constraints.

The wide range of diverse phenomenological effects that modified gravity models imprint on gravitating systems at different scales - from the dynamics of the Solar System to the expansion of the Universe and the formation LSSs (see e.g. Lombriser 2014, for an excellent recent review on observational constraints on Chameleon modified gravity theories) - provide several complementary ways to constrain deviations from standard GR. In particular, $f(R)$ gravity is already quite tightly constrained by the properties of small-scale structures, from the size of the Solar System (see e.g. Hu \& Sawicki 2007; Lombriser, Koyama \& Li 2014) to that of dwarf galaxies (see e.g. Jain \& VanderPlas 2011; Jain, Vikram \& Sakstein 2013; Vikram et al. 2013), with upper bounds on the scalar amplitude $\left|\bar{f}_{\mathrm{R}, 0}\right|$ of $8 \times 10^{-7}$ and $1 \times 10^{-7}$, respectively. Despite these very tight constraints, $f(R)$ gravity models with larger $\left|\bar{f}_{\mathrm{R}, 0}\right|$ values still remain an interesting target for LSS phenomenology, as constraints on larger scales are significantly looser (see e.g. Lombriser 2014; $\mathrm{Hu}$ et al. 2016), and since including massive neutrinos in the LSS modelling significantly loosens the bounds (Baldi et al. 2014). Furthermore, simple extensions of the basic $f(R)$ scenario - e.g. by considering an effective decoupling of the scalar field from ordinary baryonic matter - would evade the tightest local bounds and be mostly constrained by LSS formation.

In addition to what already stated, potentially new constraints on $\bar{f}_{\mathrm{R}, 0}$ may be provided by the kinematic Sunyaev-Zel'dovich (kSZ) effect of the LSS, i.e. the Doppler-shift induced on the cosmic microwave background (CMB) photons by the motion of free electrons along the line of sight. In fact, the $\mathrm{kSZ}$ effect is expected to receive significant contribution by high-redshift gas and is affected by motions on all physical scales (see e.g. Roncarelli et al. 2007; Battaglia et al. 2010; Shaw, Rudd \& Nagai 2012; Roncarelli, VillaescusaNavarro \& Baldi 2017), thus being potentially influenced by any type of modification of the gravitational force. This becomes particularly interesting after the first measurement of the amplitude of kSZ-driven temperature fluctuations at $\ell=3000$ achieved by the South Pole Telescope (SPT) team. Due to the combination of the thermal Sunyaev Zel'dovich (tSZ) effect bispectrum from the SPTSZ survey $\left(800 \mathrm{deg}^{2}\right.$ ) with the full $2540 \mathrm{deg}^{2}$ SPT field, George et al. (2015) obtained a $>2 \sigma$ detection of the $\mathrm{kSZ}$ power spectrum amplitude, i.e. $\mathcal{D}_{3000}^{\mathrm{kSZ}}=(2.9 \pm 1.3) \mu \mathrm{K}^{2}$ (see also a previous measurement in a smaller area by Crawford et al. 2014). Considering that the LSS plasma at the epoch of reionization (EoR) is expected to provide a significant, albeit uncertain, contribution to the kSZ power (see e.g. Iliev et al. 2007; Battaglia et al. 2013; Iliev et al. 2014, and references therein), this value is sufficiently low to provide meaningful constraints on reionization models, despite the relatively large errors. In our previous work (Roncarelli et al. 2017) using hydrodynamical simulations we predicted a $\mathrm{kSZ}$ power spectrum amplitude of $\mathcal{D}_{3000}^{\mathrm{kSZ}}=4.0 \mu \mathrm{K}^{2}$ from the ionized Universe in the lambda cold dark matter $(\Lambda \mathrm{CDM})$ model (assuming cosmological parameters from Planck Collaboration XIII 2016). This translates into an upper limit on the contribution form 'patchy' reionization of $\mathcal{D}_{3000}^{\mathrm{kSZ} \text {,patchy }}<1.0 \mu \mathrm{K}^{2}$ (95 per cent C.L.), which is interestingly close to conservative predictions (see e.g. Park et al. 2013) and, possibly, enough to rule out some of the most extreme models (see e.g. Mesinger, McQuinn \& Spergel 2012; Mesinger, Ferrara \& Spiegel 2013). In this scenario, modified gravity models are expected to increase the gas peculiar velocities with respect to the GR model, thus boosting the kSZ power, and making it a new and potentially competitive probe. 
This paper is the second in a series of works (following Roncarelli et al. 2017, Paper I, hereafter, on the effect of massive neutrinos) aimed at describing the properties of the kSZ effect from the LSS in different cosmological models beyond the standard $\Lambda \mathrm{CDM}$. Here, we present the first analysis of the $\mathrm{kSZ}$ effect signal derived from a set of cosmological hydrodynamical simulations that account for the effect of modified gravity with a state-of-the-art numerical code (MG-GADGET, Puchwein, Baldi \& Springel 2013). Starting from the physical properties of the baryons in the simulations, we derive the kSZ signal by constructing Doppler $b$-parameter maps integrated in the past light-cone from $z=0$ down to the EoR, and compute the amplitude of the power spectrum $(1000<\ell<20000)$ in the standard GR case and assuming different values of $\bar{f}_{\mathrm{R}, 0}$. We also study the degeneracy between $z_{\text {re }}$ (the redshift at which reionization occurs) and $\bar{f}_{\mathrm{R}, 0}$ and, by comparing our predictions with the results of George et al. (2015), we derive upper limits on $\mathcal{D}_{3000}^{\mathrm{kSZ} \text {,patchy }}$ in different modified gravity scenarios.

This manuscript is organised as follows. In the next section, we review the main definitions of modified gravity adopted for our work. In Section 3, we describe our simulations and our modelling of the kSZ effect. We discuss in Section 4 our results and draw our conclusions in Section 5.

\section{BASIC DEFINITIONS OF MODIFIED GRAVITY THEORY}

Here we review the main definitions of the modified gravity formalism adopted in our work, together with the definition of $\bar{f}_{\mathrm{R}, 0}$. For a more detailed description of this class of models, we refer he reader to some excellent reviews on the subject (De Felice \& Tsujikawa 2010; Sotiriou \& Faraoni 2010).

As stated above, in order to pass observational tests and reproduce the observed expansion history the choice of the functional form of $f(R)$ must fulfil some specific constraints. The most widely studied case for such constrained $f(R)$ functions is represented by the form (Hu \& Sawicki 2007):

$f(R)=-m^{2} \frac{c_{1}\left(\frac{R}{m^{2}}\right)^{n}}{c_{2}\left(\frac{R}{m^{2}}\right)^{n}+1}$,

where $m^{2} \equiv H_{0}^{2} \Omega_{\mathrm{M}}$ is a mass scale while $c_{1}, c_{2}$, and $n$ are nonnegative constant free parameters of the model. The choice of equation (3) has the appealing feature of allowing to recover with arbitrary precision the expansion history of a $\Lambda$ CDM cosmology by choosing $c_{1} / c_{2}=6 \Omega_{\Lambda} / \Omega_{\mathrm{M}}$ under the condition $c_{2}\left(R / m^{2}\right)^{n} \gg 1$, so that the scalar field $f_{R}$ takes the approximate form:

$f_{R} \approx-n \frac{c_{1}}{c_{2}^{2}}\left(\frac{m^{2}}{R}\right)^{n+1}$.

In this work we will restrict our analysis to models with $n=1$, so that $c_{2}$ remains the only free parameter which can be also expressed in terms of the mean value of the scalar degree of freedom at the present epoch, $\bar{f}_{R 0}$. We will then define our $f(R)$ cosmologies by their $\bar{f}_{R 0}$ value in the following.

In $f(R)$ gravity, the dynamical gravitational potential $\Phi$ corresponding to the time-time metric perturbation obeys the equation (Hu \& Sawicki 2007; Winther et al. 2015):

$\nabla^{2} \Phi=\frac{16 \pi G}{3} a^{2} \delta \rho-\frac{1}{6} a^{2} \delta R$,

which can be rewritten as:

$\nabla^{2} \Phi=\nabla^{2} \Phi_{\mathrm{N}}-\frac{1}{2} \nabla^{2} f_{R}$
Table 1. Parameter values of our set of simulations. First column: simulation name. Second column: comoving box size, in $h^{-1} \mathrm{Mpc}$. Third column: total number of particles (DM + baryons). Fourth and fifth column: masses of the DM and baryonic particles, respectively, in units of $10^{9} h^{-1} M_{\odot}$. Sixth column: value of $\bar{f}_{\mathrm{R}, 0}$. All simulations assume a flat cosmology with $\Omega_{\Lambda}=$ $0.6866, \Omega_{\mathrm{m}}=\Omega_{\mathrm{CDM}}+\Omega_{\mathrm{b}}=0.3134, \Omega_{\mathrm{b}}=0.049, H_{0}=67 \mathrm{~km} \mathrm{~s}^{-1} \mathrm{Mpc}^{-1}$ ( $h=0.67$ ), $n_{s}=0.96$, and $A_{s}=2.13 \times 10^{-9}$ (corresponding to $\sigma_{8}=0.834$ at $z=0$ for the GR simulation). The physical scheme assumed for the baryonic component includes radiative cooling, UV background, and star formation following the 'quick-Lyman- $\alpha$ ' method (see text for details). These parameters are defined so that the GR simulation matches the NO simulation of Paper I.

\begin{tabular}{lccccc}
\hline Simulation & $\begin{array}{c}L_{\text {box }} \\
\left(h^{-1} \mathrm{Mpc}\right)\end{array}$ & $N_{p}$ & $\begin{array}{c}m_{p, \mathrm{cdm}} \\
\left(10^{9} h^{-1} M_{\odot}\right)\end{array}$ & $m_{p, \mathrm{~b}}$ \\
\hline GR & 240 & $2 \times 512^{3}$ & 7.56 & 1.40 & 0 \\
FR-6 & 240 & $2 \times 512^{3}$ & 7.56 & 1.40 & $-10^{-6}$ \\
FR-5 & 240 & $2 \times 512^{3}$ & 7.56 & 1.40 & $-10^{-5}$ \\
FR-4 & 240 & $2 \times 512^{3}$ & 7.56 & 1.40 & $-10^{-4}$ \\
\hline
\end{tabular}

where $\Phi_{\mathrm{N}}$ is the standard Newtonian potential. From equation (6) it follows that the total gravitational force in $f(R)$ gravity is dictated by a modified potential $\Phi=\Phi_{\mathrm{N}}-\frac{1}{2} f_{R}$ (while the lensing potential $\Psi$ - i.e. the space-space perturbation of the metric tensor - is not affected by the modification of gravity).

The fifth-force - proportional to $\vec{\nabla} f_{R}$ - is suppressed for scales larger than the Compton wavelength of the field, which is given by

$\lambda_{\mathrm{C}}=a^{-1} \sqrt{3 \mathrm{~d} f_{R} / \mathrm{d} R}=a^{-1} \sqrt{6\left|f_{R 0}\right| \frac{\bar{R}_{0}^{2}}{R^{3}}}$.

This sets a maximum scale for the effects of the modification of gravity, thereby introducing a scale dependence in the model. Additionally, due to the non-linear dependence of $\delta R$ on $f_{R}$, the theory exhibits the so-called Chameleon screening mechanism (Khoury \& Weltman 2004): the Compton wavelength $\lambda_{\mathrm{C}}$ shrinks (or equivalently the scalar field mass $m_{f_{R}} \sim 1 / \lambda_{\mathrm{C}}$ increases) in high-density environments, where the fifth-force propagation is then confined to arbitrarily short distances. This provides a mechanism to evade Solar System constraints on gravity (see e.g. Bertotti et al. 2003; Lombriser 2014; Will 2006)

\section{MODELLING THE EFFECT OF $f(R)$ ON THE KSZ SIGNAL}

\subsection{The hydrodynamical simulations}

As mentioned above, for our simulations we have used the MGGADGET code (Puchwein et al. 2013), a modified version of GADGETIII (Springel 2005) that includes the effects of the modified potential and its associated Chameleon screening mechanism. MG-GADGET solves equation (2) for a generic density field produced by a set of discrete particles by means of a Newton-Gauss-Seidl iterative scheme, and computes the total force on each particle via equation (5) by including the curvature perturbation $\delta R$ (derived according to equation 2) in the gravitational source term. We refer the interested reader to the MG-GADGET code paper (Puchwein et al. 2013) for an extended presentation of the numerical implementation.

In this work we will consider four different hydrodynamical simulations, whose characteristics are summarized in Table 1. The parameters have been chosen to mimic exactly the configuration of our first simulation set of Paper I (dubbed N0, N15, N30, and N60) 
on the effect of massive neutrinos, to allow for a direct comparison. Besides the GR simulation that corresponds to the standard $\Lambda \mathrm{CDM}$ scenario $\left(\bar{f}_{\mathrm{R}, 0}=0\right)$, we run three other simulations, dubbed FR-6, FR-5, and FR-4, with $\bar{f}_{\mathrm{R}, 0}=\left(-10^{-6},-10^{-5},-10^{-4}\right)$, respectively. While the largest of these values is already ruled out (see Section 1) by other probes (even though it was never tested before using the $\mathrm{kSZ}$ effect alone, as we will do in our analysis) the other models considered in this work are still marginally consistent with the most recent observational constraints and are widely employed by the community to test $f(R)$ gravity phenomenology.

Our simulations evolve a periodic distribution of $512^{3} \mathrm{CDM}$ particles and an equal number of baryonic particles, in a cosmological box of $240 h^{-1} \mathrm{Mpc}$ per side, from a starting redshift of $z_{\mathrm{i}}=99$. Initial conditions have been generated by displacing particles from a homogeneous cartesian grid according to the Zel'dovich approximation (Zel'dovich 1970) in order to obtain a random realization of the linear matter power spectrum computed with the public Boltzmann code CAMB (Lewis, Challinor \& Lasenby 2000) for the set of cosmological parameters summarized in the caption of Table 1. As the effects of Modified Gravity are fully screened in the highredshift Universe by the high background density, no modification is needed in such procedure to generate initial conditions for the $f(R)$ gravity simulations. Thus, all our simulations use exactly the same starting configuration at $z_{\mathrm{i}}=99$, and evolve according to different gravity theories from then on. CDM particles are treated as a collisionless fluid, conversely the thermo- and hydrodynamics of baryons is modelled through the Smoothed Particle Hydrodynamics (SPH) scheme included in GADGET-III. Besides adiabatic hydrodynamical forces, the treatment of SPH particles employs the simplified 'quick-Lyman- $\alpha$ ' approach (Viel et al. 2005) that converts every gas particle with density contrast above 1000 and temperature below $10^{5} \mathrm{~K}$ into a collisionless star particle. Such treatment is much less numerically demanding compared to more sophisticated implementations of star formation and feedback mechanisms, but is largely sufficient for the purposes of this work.

Since it does not depend directly on gas temperature (see details in Section 3.3), the kSZ effect of the ionized Universe is weakly dependent by the details of the feedback mechanisms affecting the baryonic component (see e.g. Roncarelli et al. 2007; Trac, Bode \& Ostriker 2011; Shaw et al. 2012, and discussions therein), unlike, for instance, the X-ray emission or the tSZ effect. When modelling the kSZ effect with hydrodynamical simulations one has to face two main challenges: (i) accounting for the amount of baryons that does not reside in the diffuse ionized state (mainly stars) and (ii) sampling a cosmological volume big enough to sample the large-scale velocity modes. In this work, we use the same approach adopted in Paper I: we refer the interested reader to its section 4 for the details of the implementation and for the relative tests with specific hydrodynamical simulations used to quantify the systematics. We summarize here the main points.

(i) We compute the star mass fraction as a function of redshift in our simulation volumes, $f_{\star, \operatorname{sim}}(z)$, shown in Fig. 1. Since our cooling scheme is known to overpredict this quantity, we take as a reference the cosmological star mass fraction, $f_{\star \text { obs }}(z)$, derived by Ilbert et al. (2013) with UltraVISTA data and correct the density of our SPH particles in the following way:

$\rho_{\mathrm{g}}(z)=\rho_{\mathrm{g}, \operatorname{sim}}(z)\left[\frac{1-f_{\star, \mathrm{obs}}(z)}{1-f_{\star, \operatorname{sim}}(z)}\right]$.

As it can be seen from the plot in Fig. 1, this corresponds to correcting the density by $\sim 1$ per cent at $z=3$ and $\sim 5$ per cent at

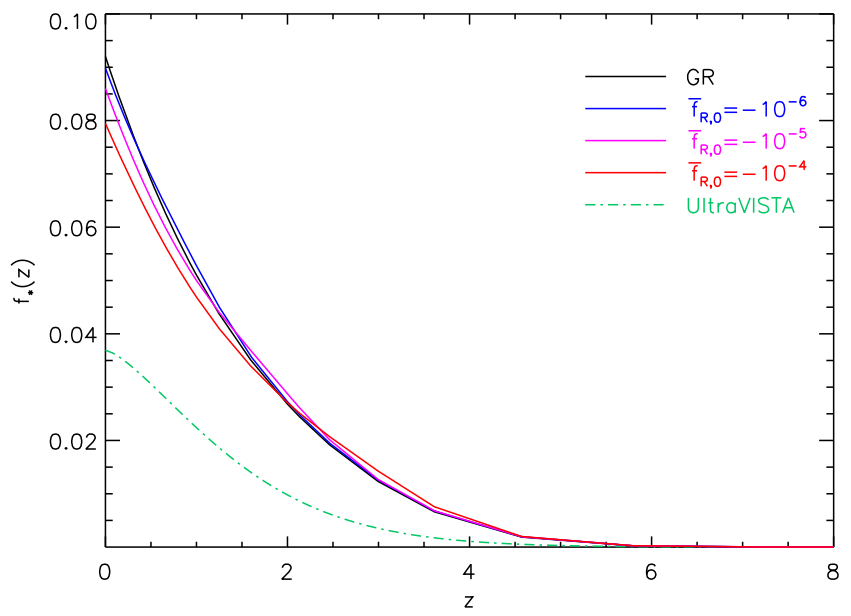

Figure 1. Global star mass fraction $\left(\rho_{\star} / \rho_{\mathrm{b}}\right)$ in the whole box volume as a function of redshift for our set of four simulations (solid lines), with $\bar{f}_{\mathrm{R}, 0}=\left(0,-10^{-6},-10^{-5},-10^{-4}\right)$ shown in black, blue, magenta, and red, respectively. The green dot-dashed line is derived from the cosmic stellar mass density estimated by Ilbert et al. (2013).

$z=0$. We have shown in Paper I that this approach allows to correct the $\mathrm{kSZ}$ signal mimicking the desired star formation history without introducing significant systematics (less than 5 per cent at $\ell<5000$ ).

(ii) Given the limited volume of our simulations, we need to correct for the missing velocity power on scales larger than our box size of $240 h^{-1} \mathrm{Mpc}$. By adopting an approach similar (albeit slightly more conservative) to Iliev et al. (2007), we have shown in Paper I (Section 4) that we need to increase the amplitude of the kSZ power computed from our simulations by 25 per cent ${ }^{2}$ in order to account for the missing velocity power. Since this paper has its main focus in studying the impact of modified gravity on the kSZ effect, we stress that the results presented here on the relative difference between our four simulations do not depend on this correction. However, this point clearly becomes crucial when comparing our results with SPT data, as we do in Section 4.

\subsection{Light-cone construction}

The study of the kSZ properties requires a full light-cone reconstruction, accounting for the integrated signal up to the EoR (see Section 3.3). Here, we review our method described also in Paper I and already adopted in our previous works (Roncarelli et al. 2006, 2007, 2010, 2012; Roncarelli, Carbone \& Moscardini 2015).

We stack the simulation outputs along the line of sight up to $z=15$, enough to account for the most conservative upper limits in $z_{\mathrm{re}}$. With our cosmology, this corresponds to a comoving distance of $7022 h^{-1} \mathrm{Mpc}$. The positions of the SPH particles inside each volume are randomized to avoid the repetition of the same structure inside the field of view (see details in Paper I). The randomization process is implemented using the same random seed for all simulations. Since they also share the phases of the initial conditions, this ensures that the volumes enclosed by the light-cones reproduce an identical realization of the same structures, albeit with different

\footnotetext{
${ }^{2}$ In Paper I we quoted the value of 20 per cent, referring to the missing power with respect to the total one. This translates into multiplying the simulation result by $1 /(1-0.2)=1.25$, thus increasing it by 25 per cent.
} 
$\bar{f}_{\mathrm{R}, 0}$, thus eliminating the effect of cosmic variance in the relative differences. By varying the initial random seed, we generate 50 light-cone realizations for each simulation: this allows to enhance the statistical robustness of our final results.

\subsection{The kSZ effect model}

The kSZ effect (Sunyaev \& Zeldovich 1970; Ostriker \& Vishniac 1986; Vishniac 1987) is the Doppler shift in the CMB spectrum induced by the peculiar velocity of the free electrons of the LSS. Unlike for its thermal counterpart, the shift, $\Delta T$, in the observed CMB temperature is the same at all frequencies: for a given direction, identified by the unit vector $\hat{\gamma}$, one has

$$
\Delta T(\hat{\gamma})=-b(\hat{\gamma}) T_{\mathrm{CMB}},
$$

where $T_{\mathrm{CMB}}$ is the $\mathrm{CMB}$ temperature and $b(\hat{\gamma})$ is the Doppler $b$ parameter, defined as

$b(\hat{\boldsymbol{\gamma}}) \equiv \frac{\sigma_{\mathrm{T}}}{c} \int_{0}^{z_{\mathrm{re}}} \frac{\mathrm{d} \chi}{\mathrm{d} z} \frac{\mathrm{d} z}{1+z} e^{-\tau(z)} n_{\mathrm{e}} \boldsymbol{v}_{\mathrm{e}} \cdot \hat{\boldsymbol{\gamma}}$.

In the previous equation $c$ is the vacuum light speed and $\sigma_{\mathrm{T}}$ the Thomson cross-section. The intergalactic medium (IGM) physical properties enter in the integral along the comoving coordinate $\chi$, with $n_{\mathrm{e}}$ and $\boldsymbol{v}_{\mathrm{e}}$ being the number density and the proper velocity of the electrons, respectively. Finally, the Thomson optical depth, $\tau$, is given by:

$\tau(z) \equiv \sigma_{\mathrm{T}} \int_{0}^{z} \frac{\mathrm{d} \chi}{\mathrm{d} z^{\prime}} \frac{\mathrm{d} z^{\prime}}{1+z^{\prime}} n_{\mathrm{e}}\left(z^{\prime}\right)$.

As already done in Paper I, in order to compute equation (10) from our GADGET-III simulations, we apply the following equation:

$b(\hat{\gamma})=\frac{\sigma_{\mathrm{T}} X x_{\mathrm{e}}}{c m_{\mathrm{p}}} \int_{0}^{z_{\mathrm{re}}} \frac{\mathrm{d} \chi}{\mathrm{d} z} \frac{\mathrm{d} z}{1+z} e^{-\tau(z)} \rho_{\mathrm{g}} \boldsymbol{v}_{\mathrm{g}} \cdot \hat{\boldsymbol{\gamma}}$,

where $X=0.76$ is the cosmological hydrogen mass fraction, $x_{\mathrm{e}}$ $\simeq 1.16$ is the electron-to-proton ratio and $m_{\mathrm{p}}$ the proton mass. As said in Section 3.1, to account for the fraction of baryons that are not ionized, we correct the value of the gas mass density, $\rho_{\mathrm{g}}$, with equation (8).

\subsection{The mapping procedure}

The kSZ physical model described in Section 3.3 and the light-cone geometry defined in Section 3.2 are employed to create a set of $b$-parameter maps that are then used to compute our results. Here, we summarize the main points of our method.

For a given SPH particle, the physical variables provided by MGGADGET that are relevant for the kSZ effect are its mass, $m_{\mathrm{i}}$, its peculiar velocity, $\mathbf{v}_{\mathbf{i}}$, and its smoothing length, $h_{\mathrm{i}}$. In addition, its $3 \mathrm{D}$ position in the light-cone determines its sky coordinate $\hat{\gamma}_{i}$ that is used both to place it in the map and to determine its radial velocity component $v_{r, i} \equiv \mathbf{v}_{\mathbf{i}} \cdot \hat{\gamma}_{\mathbf{i}}$. After selecting all the particles whose 'SPH-sphere', i.e. the sphere with radius $h_{\mathrm{i}}$, intersects the lightcone, their integrated $b$-parameter is computed with the following formula:

$B_{i}=\frac{X \sigma_{\mathrm{T}} x_{\mathrm{e}}}{m_{\mathrm{p}} c d_{A, i}^{2}} e^{-\tau\left(z_{i}\right)} m_{i} v_{r, i}$,

$d_{A, i}$ being the angular diameter distance from the observer, and $z_{i}$ the corresponding cosmological redshift, used to compute $\tau\left(z_{i}\right)$ with equation (11). Finally, equation (12) is converted into a discrete sum over all the values of $B_{i}$ that are distributed in the map pixels. To this purpose we take advantage of the mapping procedure defined in Ursino, Galeazzi \& Roncarelli (2010) that exploits two mathematical properties of the SPH smoothing kernel: first, the variables of the 2D integral are separated assuming that the kernel approximates a Gaussian function, then, since the kernel is a polynomial, each 1D integral is computed analytically. This ensures both the accuracy and the computational performance of the calculation. The interested reader may refer to section 3 of Ursino et al. (2010) for the details of the implementation.

For our purposes, we compute maps of $1.8^{\circ}$ per side: at $z=15$ the corresponding transverse comoving distance is $221 h^{-1} \mathrm{Mpc}$, so that our box size of $240 h^{-1}$ Mpc fully encloses the field of view. Each map is 1024 pixels per side, corresponding to an angular resolution of 6.33 arcsec. The mapping procedure is repeated for the 50 light-cones and for the full set of simulations. Moreover, we compute separately the contribution to each $b$-parameter map into 20 logarithmically equi-spaced redshift bins, by varying the limits in the integral of equation (12). On the whole this makes a total of $1000 b$-parameter maps for each simulation.

\section{RESULTS}

\subsection{Global properties of the Doppler $b$-parameter}

We show in Fig. 2 the $b$-parameter maps for our four simulations with $\bar{f}_{\mathrm{R}, 0}=\left(0,-10^{-6},-10^{-5},-10^{-4}\right)$. The maps have been obtained from the same light-cone and considering $z_{\mathrm{re}}=8.8$, i.e. the nominal value as measured by Planck Collaboration XIII (2016). Coherently with our analysis of Paper I, the typical values are of the order of $|b| \approx 10^{-6}$, enough to induce CMB temperature increments or decrements of several $\mu \mathrm{K}$ (see equation 9). The map peaks, associated to galaxy clusters, can reach values an order-of-magnitude larger. The larger modes of the size of $\sim 10$ arcmin are connected to coherent motions in the LSS.

The effect of the different values of $\bar{f}_{\mathrm{R}, 0}$ is not evident by eye. In order to highlight these differences, we show in Fig. 3 the distribution of pixel values computed for the four models from the full 50 light-cones (i.e. a total of $5.2 \times 10^{7}$ pixels each). It is clear that the effect of $f(R)$ models is to increase the chance of larger $b$-parameter values: qualitatively this is expected, since the enhancement of the gravitational force with respect to the standard GR model has a direct impact on the velocity field. The effect increases for larger absolute values of $\bar{f}_{\mathrm{R}, 0}$.

We fit all the four distributions with a Gaussian curve, with fixed normalization and with mean and dispersion, $\sigma_{b}$, as free parameters. The results for the values of $\sigma_{b}$, shown in the top right corner of Fig. 3, range from $1.27 \times 10^{-6}$ for the GR model to $1.43 \times 10^{-6}$ for $\bar{f}_{\mathrm{R}, 0}=-10^{-4}$. We have verified empirically that the scaling of $\sigma_{b}$ with $\bar{f}_{\mathrm{R}, 0}$ well follows a power-law form of this type:

$\sigma_{b, \bar{f}_{\mathrm{R}, 0}}=\sigma_{b, \mathrm{GR}}\left(1+\sqrt{\left|\bar{f}_{\mathrm{R}, 0}\right|}\right)^{12.5}$,

where $\sigma_{b, \mathrm{GR}}$ is the value for the GR model. As we will show in Section 4.2, the dependence with $\left(1+\sqrt{\left|\bar{f}_{\mathrm{R}, 0}\right|}\right)$ does not describe only the scaling of $\sigma_{b}$, but works well also to trace the variations of the kSZ power spectrum amplitude. Since this is the attempt to define the scaling of an observational quantity in $f(R)$ models, a possible interesting test would be to verify if a formula of this type works also with other important physical or observational quantities, such as the halo mass function or the tSZ effect power spectrum. 

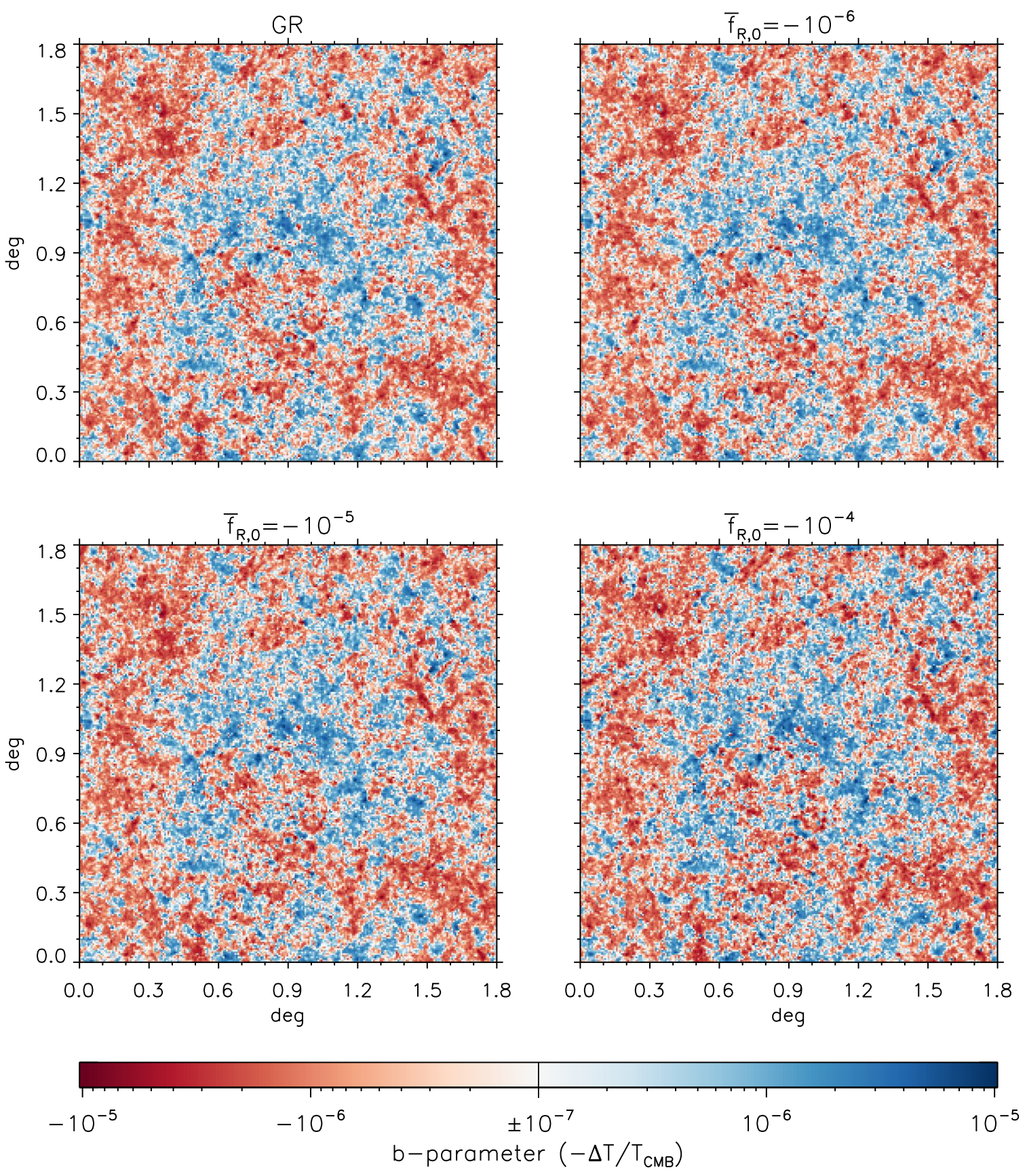

Figure 2. Maps of the $b$-parameter as a function of $\bar{f}_{\mathrm{R}, 0}$. Each map is $1.8^{\circ}$ per side with a resolution of 6.33 arcsec (1024 pixels) and represents the signal integrated from $z=0$ to $z_{\mathrm{re}}=8.8$ for the same light-cone assuming general relativity $\left(\bar{f}_{\mathrm{R}, 0}=0\right.$, top-left) and $\bar{f}_{\mathrm{R}, 0}=\left(-10^{-6},-10^{-5},-10^{-4}\right)$ (top-right, bottom-left, and bottom-right, respectively). The colour scale indicates in red the sky regions where, on average, the gas is approaching the observer $(b<0$ and increase of observed CMB temperature), and in blue where the gas is receding ( $b>0$ and decrease of observed CMB temperature).

Since this goes beyond the scope of this paper, we leave this for future works.

\subsection{The $\mathrm{kSZ}$ effect power spectrum}

In order to compare our results with the SPT measurement by George et al. (2015) we need to calculate the amplitude of the angular power spectrum of temperature fluctuations induced by the $\mathrm{kSZ}$ effect as a function of the multipole $\ell$. We do so by converting our $b$-parameter into $\Delta T$ maps with equation (9) and by applying a Fast Fourier Transform in the flat-sky approximation (see the detailed explanation in Roncarelli et al. 2007). Following Crawford et al. (2014), we express the results in terms of

$\mathcal{D}_{\ell} \equiv \frac{\ell(\ell+1) C_{\ell}}{2 \pi}$,

where $C_{\ell}$ is the definition of power spectrum of temperature fluctuations normally adopted in CMB analyses.

The results of the $\mathrm{kSZ}$ power spectrum amplitude as a function of $\ell$ for our four models and assuming $z_{\text {re }}=8.8$ are shown in Fig. 4 (solid lines). Overall the shape of the power spectrum is the same for all models, with an increase from $\ell=1000$ to 6000 followed by 


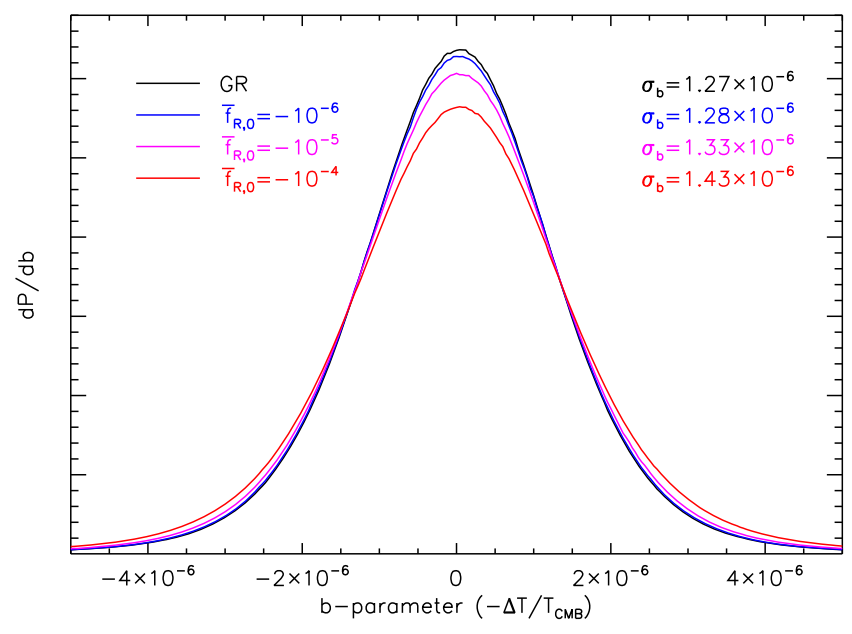

Figure 3. Probability distribution function of the $b$-parameter for different values of $\bar{f}_{\mathrm{R}, 0}$. Each curve is computed for the whole set of 50 light-cones (i.e. 50 sets of maps like the ones shown in Fig. 2) with signal integrated up to $z_{\text {re }}=8.8$ and pixels of 6.33 arcsec per side. The colour coding is the same as in Fig. 1. On the top right corner we show the standard deviation of the best-fitting Gaussian distribution of each curve.

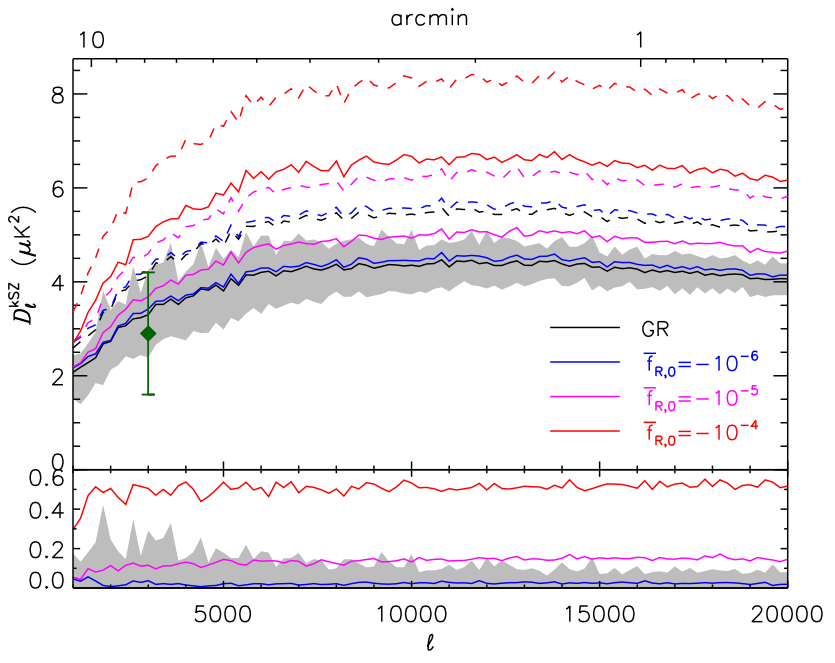

Figure 4. Top panel: angular power spectrum of temperature fluctuations associated with the $\mathrm{kSZ}$ effect $\left(\mathcal{D}_{\ell}^{\mathrm{kSZ}}\right)$ as a function of the multipole $\ell$ for different values of $\bar{f}_{\mathrm{R}, 0}$. The curves are computed by averaging over the full set of 50 light-cones (a total of $162 \mathrm{deg}^{2}$ ) and consider the signal integrated up to $z_{\mathrm{re}}=8.8$. The colour coding is the same as in Fig. 1. The grey-shaded area encloses the central 34 light-cones (68 per cent of the total) for the GR model $\left(\bar{f}_{\mathrm{R}, 0}=0\right)$ only. The solid lines indicate values derived directly from the maps, dashed lines account for the 20 per cent correction due to the limited box size. The green diamond with error bars shows the measurement of $\mathcal{D}_{3000}^{\mathrm{kSZ}}=(2.9 \pm 1.3) \mu \mathrm{K}^{2}$ by George et al. (2015). Bottom panel: relative differences with respect to the GR model.

an almost flat 'plateau': this is consistent with our previous results in Paper I and in Roncarelli et al. (2007). As expected, the effect of modified gravity is to boost the $\mathrm{kSZ}$ power: this enhancement is 2-3 per cent for the FR-6 model, 10-15 per cent for the FR-5, up to about 50 per cent for the FR-4. Interestingly, the effect of $f(R)$ appears to be scale-independent, i.e. the relative differences do not depend on $\ell$. On the other hand, the effect of massive neutrinos discussed in Paper I showed a clear dependence on the angular scale, decreasing in intensity for larger $\ell$, thus providing a poten- tial method to remove the degeneracies between the two effects. In fact, it is now well known that $f(R)$ gravity and massive neutrinos are strongly degenerate with each other, so that specific combinations of their characteristic parameters (namely $\bar{f}_{\mathrm{R}, 0}$ and $\Sigma m_{v}$ ) may be hardly distinguishable from the standard $\Lambda \mathrm{CDM}$ model for a wide range of basic cosmological observables (see e.g. He 2013; Motohashi, Starobinsky \& Yokoyama 2013; ; Baldi et al. 2014; Wright, Winther \& Koyama 2017) and more sophisticated statistics are needed to disentangle their effects (see e.g. the recent outcomes of Peel et al. 2018).

Apart from relative differences, a comparison with observational results requires to apply the correction due to the limited simulation volume detailed in Section 3.1, that we estimate in an increase of 25 per cent. The corresponding curves with this corrections are shown with dashed lines in Fig. 4. Most importantly, we predict a value of $\mathcal{D}_{3000}^{\mathrm{kSZ}}=4.1 \mu \mathrm{K}^{2}$ for the $\Lambda \mathrm{CDM}$-GR model, consistent with our previous result of $=4.0 \mu \mathrm{K}^{2}$ in Paper I (N0 run, volume corrected), enough to account for all of the signal of $\mathcal{D}_{3000}^{\mathrm{kSZ}}=(2.9 \pm 1.3) \mu \mathrm{K}^{2}$ measured by George et al. (2015), and close to its $+1 \sigma$ limit, even without including the contribution from patchy reionization. We discuss the implications of our result on these type of models in Section 4.4. As expected, our three modified gravity models predict larger values, with $\mathcal{D}_{3000}^{\mathrm{kSZ}}=(4.3,4.6,6.2) \mu \mathrm{K}^{2}$ for $\bar{f}_{\mathrm{R}, 0}=\left(-10^{-6}\right.$, $\left.-10^{-5},-10^{-4}\right)$, respectively. Only the $\bar{f}_{\mathrm{R}, 0}=-10^{-4}$ model is ruled out at a significant level $(2.5 \sigma)$, indicating that differences associated with realistic $f(R)$ model would be difficult to measure with current CMB instruments.

The predictions on the kSZ effect observables depend on the cosmological assumptions (Shaw et al. 2012). Therefore, our results on $\bar{f}_{\mathrm{R}, 0}$ are degenerate with respect to the standard cosmological parameters and with the value of the neutrino mass fraction, $f_{v}$, as studied in Paper I. With our set of data it is easy to show the dependence of our results on the value of $z_{\text {re }}$ that, given the current measurements errors, constitutes one of the main sources of uncertainty.

Fig. 5 shows the values of $\mathcal{D}_{3000}^{\mathrm{kSZ}}$ as a function of $z_{\text {re }}$ for our four simulations (solid lines) obtained by varying iteratively the upper limit in the integral of equation (12). We also show with dashed lines the volume-corrected values that can be directly compared with the SPT measurements, with uncertainties, by George et al. (2015) and the estimate of $z_{\text {re }}$ by Planck Collaboration XIII (2016) (green point with shaded region). These curves show how the kSZ effect receives significant contribution from both low- and highredshift gas, as already discussed in Paper I (see also Roncarelli et al. 2007; Shaw et al. 2012). Most notably, we observe that the differences induced by modified gravity are particularly relevant at later epochs, where non-linear effects play an important role. As an example, in the $0<z<1$ range the value of $\mathcal{D}_{3000}^{\mathrm{kSZ}}$ for the FR-4 model is double with respect to the GR one: 1.8 and $3.6 \mu \mathrm{K}^{2}$ (volume corrected), respectively. On the contrary, differences in the contribution at earlier epochs are less pronounced.

Following our analysis in Paper I, we provide a more direct estimate of the dependence with respect to $z_{\text {re }}$ and $\bar{f}_{\mathrm{R}, 0}$ by defining an analytical expression. We observe that our results can be fit with the following formula:

$$
\mathcal{D}_{\ell}^{\mathrm{kSZ}}=\mathcal{D}_{\mathrm{GR}, \ell}^{\mathrm{kSZ}}\left(\frac{z_{\mathrm{re}}}{8.8}\right)^{\alpha}\left(1+\sqrt{\left|\bar{f}_{\mathrm{R}, 0}\right|}\right)^{\gamma},
$$

where $\mathcal{D}_{\mathrm{GR}, \ell}^{\mathrm{kSZ}}$ is the result for our GR model. The best-fitting values of $\alpha$ and $\gamma$ for the different multipoles are shown in Table 2, together with the corresponding values of $\mathcal{D}_{\ell}^{\mathrm{kSZ}}$, with and without volume correction. These results confirm our previous findings of 


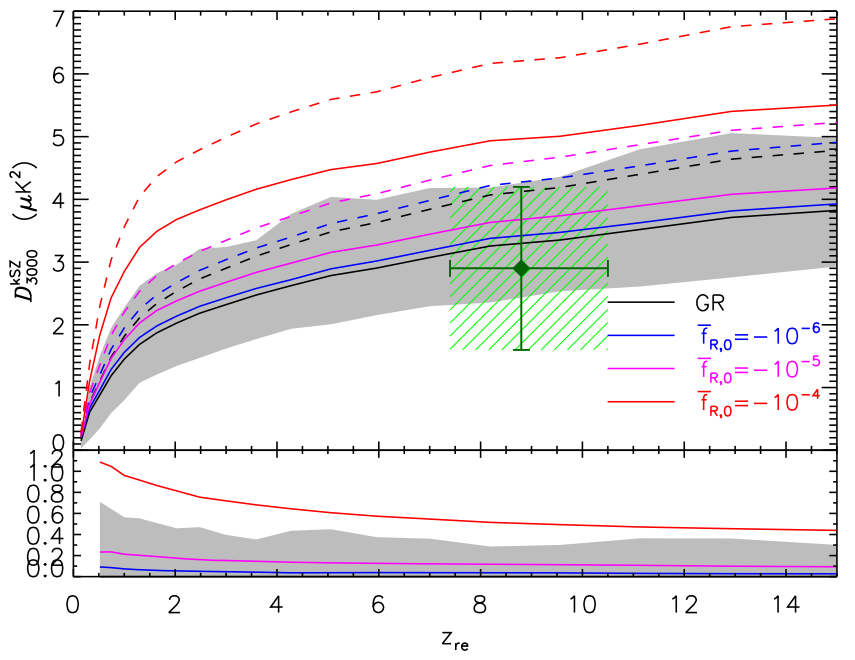

Figure 5. Top panel: amplitude of the $\mathrm{kSZ}$ power spectrum at $\ell=3000$ $\left(\mathcal{D}_{3000}^{\mathrm{kSZ}}\right)$ as a function of $z_{\text {re }}$ for different values of $\bar{f}_{\mathrm{R}, 0}$. The colour coding is the same as in Fig. 1. The grey-shaded area encloses the central 34 lightcones (68 per cent of the total) for the GR model $\left(\bar{f}_{\mathrm{R}, 0}=0\right)$ only. The solid lines indicate values derived directly from the maps, dashed lines account for the 25 per cent correction due to the limited box size. The green diamond with error bars $(1 \sigma)$ and green-shaded area shows the results of $z_{\mathrm{re}}=8.8_{-1.4}^{+1.7}$ and $\mathcal{D}_{3000}^{\mathrm{kSZ}}=(2.9 \pm 1.3) \mu \mathrm{K}^{2}$ by Planck Collaboration XIII (2016) and George et al. (2015), respectively. Bottom panel: relative differences with respect to the GR model.

Table 2. Amplitude of the $\mathrm{kSZ}$ effect power spectrum, $\mathcal{D}_{\ell}^{\mathrm{kSZ}}$, and its dependence with $z_{\text {re }}$ and $\bar{f}_{\mathrm{R}, 0}$. First column: multipole $\ell$. Second column: value of $\mathcal{D}_{\ell}^{\mathrm{kSZ}}$, in $\mu \mathrm{K}^{2}$, obtained assuming GR $\left(\bar{f}_{\mathrm{R}, 0}=0\right)$, computed averaging over the 50 light-cones ( $3.24 \mathrm{deg}^{2}$ each). Third column: $\mathcal{D}_{\ell}^{\mathrm{kSZ}}$ after applying a correction that accounts for the 25 per cent correction (i.e. 20 per cent of missing signal) due to the limited box size. The last two columns show the dependence of $\mathcal{D}_{\ell}^{\mathrm{kSZ}}$ on the redshift of reionization and $\bar{f}_{\mathrm{R}, 0}$, in terms of best-fitting values of the exponents $\alpha$ and $\gamma$ as in equation (16).

\begin{tabular}{lcccc}
\hline$\ell$ & \multicolumn{2}{c}{$\mathcal{D}_{\ell}^{\mathrm{kSZ}}\left(\mu \mathrm{K}^{2}\right)$} & $\left(\frac{z_{\mathrm{re}}}{8.8}\right)^{\alpha}\left(1+\sqrt{\left|\bar{f}_{\mathrm{R}, 0}\right|}\right)^{\gamma}$ \\
& Uncorr. & Vol. corr. & $\alpha$ & $\gamma$ \\
\hline 2000 & 2.73 & 3.4 & 0.30 & 41 \\
3000 & 3.30 & 4.1 & 0.28 & 41 \\
4000 & 3.66 & 4.6 & 0.24 & 43 \\
5000 & 3.80 & 4.8 & 0.22 & 43 \\
6000 & 4.20 & 5.2 & 0.21 & 41 \\
8000 & 4.37 & 5.5 & 0.17 & 41 \\
10000 & 4.35 & 5.4 & 0.19 & 43 \\
15000 & 4.31 & 5.4 & 0.15 & 42 \\
20000 & 4.06 & 5.1 & 0.13 & 42 \\
\hline
\end{tabular}

Paper I (see Table 2), with the values of $\mathcal{D}_{\ell}^{\mathrm{kSZ}}$ consistent in the range of a few per cent, and with almost identical values of $\alpha$. This corroborates the fact that the dependence on $z_{\text {re }}$ decreases substantially at smaller angular scales, going from $\alpha=0.30$ at $\ell=2000$, down to 0.13 at $\ell=20000$. The results on the scaling with $\bar{f}_{\mathrm{R}, 0}$ confirm in a more quantitative way to be scale independent, with scaling in the range $\left(1+\sqrt{\left|\bar{f}_{\mathrm{R}, 0}\right|}\right)^{41-43}$ with no significant trend with $\ell$. Since the scaling with $\sigma_{8}$ is expected to vary, albeit mildly, with angular scale (see e.g. Shaw et al. 2012), this suggests that the combination of $\mathcal{D}_{\ell}^{\mathrm{kSZ}}$ measurements at different $\ell$ may break the $\sigma_{8}-\bar{f}_{\mathrm{R}, 0}$ degeneracy.
Finally, by combining the results presented here with the ones of Paper I, we can provide a comprehensive formula for the scaling of the amplitude of the $\mathrm{kSZ}$ power spectrum for a combination of $f(R)$ modified gravity and massive neutrinos: ${ }^{3}$

$\mathcal{D}_{\ell}^{\mathrm{kSZ}}=\mathcal{D}_{0, \ell}^{\mathrm{kSZ}}\left(\frac{z_{\mathrm{re}}}{8.8}\right)^{\alpha}\left(1-f_{v}\right)^{\beta}\left(1+\sqrt{\left|\bar{f}_{\mathrm{R}, 0}\right|}\right)^{\gamma}$.

This expression accounts for the dependence on $z_{\text {re }}$, the neutrino mass fraction $f_{v}$ (see the values of $\beta$ in Table 2 of Paper I) and modified gravity through $\bar{f}_{\mathrm{R}, 0}$, and is valid from $\ell=2000$ to 20000 , under the assumption that the two effects are decoupled. Although the last statement seems to be supported by the tight degeneracy between the respective observational effects (see again Baldi et al. 2014; Peel et al. 2018) it was certainly not yet demonstrated for $\mathrm{kSZ}$ observations, which would require running a dedicated suite of combined hydrodynamical simulations. This goes beyond the scope of this paper, and we leave such analysis for future work.

\subsection{Comparison with the literature}

The effect of modified gravity on the kSZ power spectrum is a pioneer topic, that has not been much explored in the literature. The only work so far is by Bianchini \& Silvestri (2016), who provide a set of analytical estimates of the post-ionization $\mathrm{kSZ}$ power spectrum shape with a family of modified gravity models similar to the ones adopted here, i.e. following the Hu \& Sawicki (2007) formalism with $n=1$. A comparison of our works is therefore very interesting, in particular to test the possibile differences associated to their analytical approach, using power spectra obtained with MG-CAMB (Zhao et al. 2009) and MG-HALOFIT (Zhao 2014), with respect to our numerical method with MG-GADGET.

In agreement with our results, Bianchini \& Silvestri (2016) observe an increase of the $\mathrm{kSZ}$ that does not depend on the angular scale. This confirms that the scale dependence induced in the density and velocity power spectrum by modified gravity is washed away with the projection along the line of sight. On the other hand, while their prediction for $\bar{f}_{\mathrm{R}, 0}=-10^{-5}$ of a 10 per cent increase in $\mathcal{D}_{\ell}^{\mathrm{kSZ}}$ is consistent with our findings (magenta line in the bottom panel of Fig. 4), albeit slightly smaller, for $\bar{f}_{\mathrm{R}, 0}=-10^{-4}$ they predict a boost in $\mathcal{D}_{\ell}^{\mathrm{kSZ}}$ by 30 per cent against our 50 per cent (red line). This discrepancy is likely an indication that Bianchini \& Silvestri (2016) are underestimating the non-linear contribution of the low$z$ LSS to the kSZ effect. This is also the possible explanation of their lower value of $\mathcal{D}_{3000}^{\mathrm{kSZ}}$ for the GR model. In detail, they predict $\mathcal{D}_{3000}^{\mathrm{kSZ}} \simeq 3.85 \mu \mathrm{K}^{2}$, with cosmological parameters almost identical to ours, except for the assumption $z_{\mathrm{re}}=9.9$ instead of our more conservative $z_{\mathrm{re}}=8.8$. When adopting the same value, our prediction rises to $\mathcal{D}_{3000}^{\mathrm{kSZ}}=4.2 \mu \mathrm{K}^{2}\left(z_{\mathrm{re}}=9.9\right.$, volume corrected $)$, thus about 10 per cent higher.

Regarding this point, in a recent work Park et al. (2016) provided a detailed study on the importance of including the connected four-point term in the transverse momentum power spectrum (see also Park, Alvarez \& Bond 2018). This term, which is usually neglected in analytical predictions, becomes non-negligible at wave numbers $k \gtrsim 1 \mathrm{~h} \mathrm{Mpc}^{-1}$ at low redshift, enough to contribute to about 10 per cent the of the $\mathrm{kSZ}$ effect signal of the LSS computed with the unconnected terms alone. Our numerical approach considers both the linear and non-linear regime at

\footnotetext{
${ }^{3}$ The formula that appears in equation (11) of Paper I mistakenly reports $z_{\mathrm{re}}{ }^{\alpha}$ instead of the correct $\left(\frac{z_{\mathrm{re}}}{8.8}\right)^{\alpha}$.
} 
high precision, therefore confirming the results of Park et al. (2016). We stress that this aspect is crucial when comparing $\mathrm{kSZ}$ power spectrum predictions with observational data, making our results potentially interesting to provide constraints on both cosmology and reionization models (see also the discussion in Paper I).

\subsection{Implications for patchy reionization scenarios}

Since we are considering only the contribution of the ionized IGM after the EoR, if the reionization process is non-homogeneous then the presence of ionized bubbles would produce an additional kSZ effect adding to the total value of $\mathcal{D}_{\ell}^{\mathrm{kSZ}}$. Being the expected size of these 'patches' of the order of $\sim 10 \mathrm{Mpc}$ (comoving), this would contribute in particular at $\ell=3000$, i.e. at the angular scale measured by SPT.

By combining our results for the GR simulation with the SPT measurement, we obtain an upper limit on the additional kSZ power associated to patchy reionization of $\mathcal{D}_{3000}^{\mathrm{kSZ} \text {,patchy }}<0.9 \mu \mathrm{K}^{2}$ (95 per cent C.L.), valid under the assumption of $z_{\mathrm{re}}=8.8$ and with the cosmological parameters quoted in Table 1 . This limit is very stringent and favours homogeneous and/or fast reionization scenarios. Unfortunately, only a few works on reionization models provide forecasts for the corresponding contribution to the kSZ power spectrum. Among those, the models described in Iliev et al. (2007) predict values in the range $\mathcal{D}_{3000}^{\mathrm{kSZ} \text {, patchy }} \sim 2.2-3.7 \mu \mathrm{K}^{2}$, depending on the photon production efficiency of the ionizing sources, well above the upper limits reported here. The same applies to the models of Mesinger et al. (2012), with predictions of $\mathcal{D}_{3000}^{\mathrm{kSZ} \text {,patchy }}=1.5-3.5 \mu \mathrm{K}^{2}$. Moreover, most of the models described in Mesinger et al. (2013), with $\mathcal{D}_{3000}^{\mathrm{kSZ}, \text { patchy }}=1.2-2.3 \mu \mathrm{K}^{2}$, are also ruled out. Only the model that assumes a large contribution of X-ray ionizing sources indicates a significantly smaller $\mathcal{D}_{3000}^{\mathrm{kSZ} \text {,patchy }}=0.95 \mu \mathrm{K}^{2}$, thus close to the upper limit presented in this work. On the other hand, the reionization models by Park et al. (2013) predict $\mathcal{D}_{3000}^{\mathrm{kSZ} \text {, patchy }}=0.66-0.83 \mu \mathrm{K}^{2}$ : these more conservative forecasts are below our upper limit, albeit very close.

In addition, we stress that when including $f(R)$ gravity the corresponding upper limits on $\mathcal{D}_{3000}^{\mathrm{kSZ} \text {,patchy }}$ shrink to $<0.7$ and $<0.4 \mu \mathrm{K}^{2}$ (95 per cent C.L.) for $\bar{f}_{\mathrm{R}, 0}=10^{-6}$ and $10^{-5}$, respectively. With $\bar{f}_{\mathrm{R}, 0}=10^{-4} \mathcal{D}_{3000}^{\mathrm{kSZ} \text {, patchy }}$ is consistent with zero at 99.5 per cent.

\section{SUMMARY AND CONCLUSIONS}

In this paper, we presented the first prediction of the properties of the kSZ effect of the post-reionization LSS derived from a set of hydrodynamical simulations that include the effect of $f(R)$ modified gravity. Using MG-GADGET (Puchwein et al. 2013), we run a set of four simulations in a $L_{\mathrm{box}}=240 h^{-1} \mathrm{Mpc}$ comoving box with the same cosmology but with different modified gravity parameters, namely $\bar{f}_{\mathrm{R}, 0}=\left(0,-10^{-6},-10^{-5},-10^{-4}\right)$, where $\bar{f}_{\mathrm{R}, 0}$ is defined according to the $\mathrm{Hu} \&$ Sawicki (2007) formalism and $\bar{f}_{\mathrm{R}, 0}=0$ corresponds to the standard GR case. Starting from the simulation outputs, we compute a set of 50 Doppler $b$-parameter maps derived from different light-cone realizations that we use to predict the properties of the kSZ effect signal, most notably its power spectrum, and quantify how the $\bar{f}_{\mathrm{R}, 0}$ parameter affects them. By keeping track of the line-of-sight information, we are able to provide the redshift distribution of the $\mathrm{kSZ}$ signal, allowing us to study the dependence with $z_{\text {re }}$, the redshift at which reionization occurs.
As in Roncarelli et al. (2017, our previous study on the effect of massive neutrinos, i.e. Paper I) we correct for the missing velocity power due to the limited simulation volume with a calibrated analytical formula. We also account for the cosmological star mass fraction relying on observational results (Ilbert et al. 2013). This allows us to compare our results on the kSZ effect with the recent SPT measurement of $\mathcal{D}_{3000}^{\mathrm{kSZ}}=(2.9 \pm 1.3) \mu \mathrm{K}^{2}$ by George et al. (2015) and to provide stringent upper limits on the possible contribution to the $\mathrm{kSZ}$ power by patchy reionization.

Our main findings can be summarized as follows.

(i) As in Paper I, we infer that the distribution of the Doppler- $b$ parameter can be described by a Gaussian curve, centred in 0 . Its dispersion increases for larger absolute values of $\bar{f}_{\mathrm{R}, 0}$, scaling as $\sigma_{b} \propto\left(1+\sqrt{\left|\bar{f}_{\mathrm{R}, 0}\right|}\right)^{12.5}$.

(ii) The amplitude of the kSZ power spectrum is boosted by the presence of modified gravity. The increase with respect to the GR scenario is about $2-3$ per cent for $\bar{f}_{\mathrm{R}, 0}=10^{-6}$ and rises up to 50 per cent for $\bar{f}_{\mathrm{R}, 0}=10^{-4}$, almost independent on the angular scale.

(iii) For our fiducial GR model we predict an amplitude of the $\mathrm{kSZ}$ power spectrum due to the ionized LSS at $\ell=3000$ of $\mathcal{D}_{3000}^{\mathrm{kSZ}}=4.1 \mu \mathrm{K}^{2}\left(z_{\mathrm{re}}=8.8\right)$, consistent with our findings of $\mathrm{Pa}$ per I. By combining it with SPT results (George et al. 2015), we confirm a stringent upper limit to the $\mathrm{kSZ}$ contribution due to patchy reionization of $\mathcal{D}_{3000}^{\mathrm{kSZ} \text {,patchy }}<0.9 \mu \mathrm{K}^{2}$ (95 per cent $\mathrm{C}$. L.), thus favouring homogeneous and fast reionization scenarios and ruling out many of the current models of the EoR history.

(iv) In the presence of modified gravity consistent with current constraints, these limits shrink to $\mathcal{D}_{3000}^{\mathrm{kSZ} \text {,patchy }}<0.7$ for $\bar{f}_{\mathrm{R}, 0}=10^{-6}$, and $<0.4 \mu \mathrm{K}^{2}$ (95 per cent C.L.) for $10^{-5}$.

(v) Finally, we studied the scaling of the $\mathrm{kSZ}$ power spectrum with $z_{\text {re }}$ and $\bar{f}_{\mathrm{R}, 0}$ and provide a fitting formula, equation (16), that works for $2000<\ell<20000$. The best-fitting parametres are shown in Table 2 . The scaling with $\bar{f}_{\mathrm{R}, 0}$ is approximately $\mathcal{D}_{\ell}^{\mathrm{kSZ}} \propto\left(1+\sqrt{\left|\bar{f}_{\mathrm{R}, 0}\right|}\right)^{42}$.

Our work confirms that the kSZ effect of the LSS is an extremely promising probe of the high-redshift Universe. In fact, despite the little observational data available, with an accurate modelling of the LSS after the EoR it is already possible to obtain interesting constraints on both non-standard cosmology and reionization models. We also show the importance of hydrodynamical simulations that prove to be competitive with analytical estimates.

In future we plan to extend our work with other non-standard cosmological models, such as quintessence and coupled dark energy. Since these scenarios are expected to enhance the amplitude of the kSZ power spectrum (see e.g. Ma \& Zhao 2014), this will allow to obtain further constraints on the nature of dark energy, complementary with other probes, as well as studying the degeneracies with the different cosmological parameters beyond the $\Lambda \mathrm{CDM}$ model.

\section{ACKNOWLEDGEMENTS}

This work has been completed despite the shameful situation of the Italian research system, worsened by an entire decade of severe cuts to the fundings of public universities and research institutes (see e.g. Abbott 2006, 2016, 2018). With some noticeable - yet statistically 
negligible - exceptions, this has caused a whole generation of valuable researchers, in all fields, to struggle in poor working conditions with little hope of achieving decent employment contracts and permanent positions, resulting in obvious difficulties in the planning of future research activities, and in open violation of the European Charter for Researchers. ${ }^{4}$

This work has been supported by ASI (Italian Space Agency) through the Contract n. 2015-046-R.0. MR also acknowledges financial contribution from the agreement ASI n. I/023/12/0 'Attività relative alla fase $\mathrm{B} 2 / \mathrm{C}$ per la missione Euclid'. The computational analyses have been done due to CPU time granted by the ISCRA-CINECA project 'The kinetic SZ effect as a cosmological probe'. The work of FVN is supported by the Simons Foundation. MB acknowledges support from the Italian Ministry for Education, University and Research (MIUR) through the SIR individual grant SIMCODE, project number RBSI14P4IH. We thank the anonymous referee for providing comments that helped the presentation of our work. We also thank N. Battaglia, O. Cucciati, A. Mesinger, L. Moscardini, E. Vanzella, M. Viel, and G. Zamorani for useful suggestions and discussions. We are grateful to F. Bianchini and A. Silvestri for reading the draft and providing useful comments.

\section{REFERENCES}

Abbott A., 2006, Nature, 440, 264

Abbott A., 2016, Nature, 540, 324

Abbott A., 2018, Nature, 554, 411

Abbott B. P. et al., 2017, Phys. Rev. Lett., 119, 161101

Amendola L., 2000, Phys. Rev. D, 62, 043511

Amendola L., Baldi M., Wetterich C., 2008, Phys. Rev. D, 78, 023015

Amendola L. et al., 2013, Living Rev. Relativ., 16, 6

Baker T., Bellini E., Ferreira P. G., Lagos M., Noller J., Sawicki I., 2017, Phys. Rev. Lett., 119, 251301

Baldi M., Villaescusa-Navarro F., Viel M., Puchwein E., Springel V., Moscardini L., 2014, MNRAS, 440, 75

Battaglia N., Bond J. R., Pfrommer C., Sievers J. L., Sijacki D., 2010, ApJ, 725, 91

Battaglia N., Natarajan A., Trac H., Cen R., Loeb A., 2013, ApJ, 776, 83

Bertotti B., Iess L., Tortora P., 2003, Nature, 425, 374

Bianchini F., Silvestri A., 2016, Phys. Rev. D, 93, 064026

Buchdahl H. A., 1970, MNRAS, 150, 1

Crawford T. M. et al., 2014, ApJ, 784, 143

Creminelli P., Vernizzi F., 2017, Phys. Rev. Lett., 119, 251302

Damour T., Polyakov A. M., 1994, Nuclear Phys. B, 423, 532

De Felice A., Tsujikawa S., 2010, Living Rev. Relativ., 13, 3

George E. M. et al., 2015, ApJ, 799, 177

He J.-h., , 2013, Phys. Rev. D, 88, 103523

Hinterbichler K., Khoury J., 2010, Phys. Rev. Lett., 104, 231301

Hu W., Sawicki I., 2007, Phys. Rev. D, 76, 064004

Hu B., Raveri M., Rizzato M., Silvestri A., 2016, MNRAS, 459, 3880

Ilbert O. et al., 2013, A\&A, 556, A55

Iliev I. T., Pen U.-L., Bond J. R., Mellema G., Shapiro P. R., 2007, ApJ, 660 , 933

Iliev I. T., Mellema G., Ahn K., Shapiro P. R., Mao Y., Pen U.-L., 2014, MNRAS, 439, 725

Jain B., VanderPlas J., 2011, J. Cosmol. Astropart. Phys., 10, 032

Jain B., Vikram V., Sakstein J., 2013, ApJ, 779, 39

Khoury J., Weltman A., 2004, Phys. Rev. D, 69, 044026

Laureijs R. et al., 2011, preprint (arXiv:1110.3193)
Lewis A., Challinor A., Lasenby A., 2000, ApJ, 538, 473

Lombriser L., 2014, Annalen der Physik, 526, 259

Lombriser L., Lima N. A., 2017, Phys. Lett. B, 765, 382

Lombriser L., Taylor A., 2016, J. Cosmol. Astropart. Phys., 3, 031

Lombriser L., Koyama K., Li B., 2014, J. Cosmol. Astropart. Phys., 3, 021

Ma Y.-Z., Zhao G.-B., 2014, Phys. Lett. B, 735, 402

María Ezquiaga J., Zumalacárregui M., 2017, Phys. Rev. Lett., 119, 251304

Mesinger A., McQuinn M., Spergel D. N., 2012, MNRAS, 422, 1403

Mesinger A., Ferrara A., Spiegel D. S., 2013, MNRAS, 431, 621

Motohashi H., Starobinsky A. A., Yokoyama J., 2013, Phys. Rev. Lett., 110, 121302

Nicolis A., Rattazzi R., Trincherini E., 2009, Phys. Rev. D, 79, 064036

Ostriker J. P., Vishniac E. T., 1986, ApJ, 306, L51

Park H., Shapiro P. R., Komatsu E., Iliev I. T., Ahn K., Mellema G., 2013, ApJ, 769, 93

Park H., Komatsu E., Shapiro P. R., Koda J., Mao Y., 2016, ApJ, 818, 37

Park H., Alvarez M. A., Bond J. R., 2018, ApJ, 853, 121

Peel A., Pettorino V., Giocoli C., Starck J.-L., Baldi M., 2018

Perlmutter S. et al., 1999, ApJ, 517, 565

Planck Collaboration XIII, 2016, A\&A, 594, A13

Puchwein E., Baldi M., Springel V., 2013, MNRAS, 436, 348

Ratra B., Peebles P. J. E., 1988, Phys. Rev. D, 37, 3406

Riess A. G. et al., 1998, AJ, 116, 1009

Roncarelli M., Moscardini L., Borgani S., Dolag K., 2007, MNRAS, 378 , 1259

Roncarelli M., Moscardini L., Branchini E., Dolag K., Grossi M., Iannuzzi F., Matarrese S., 2010, MNRAS, 402, 923

Roncarelli M., Cappelluti N., Borgani S., Branchini E., Moscardini L., 2012, MNRAS, 424, 1012

Roncarelli M., Carbone C., Moscardini L., 2015, MNRAS, 447, 1761

Roncarelli M., Moscardini L., Tozzi P., Borgani S., Cheng L. M., Diaferio A., Dolag K., Murante G., 2006, MNRAS, 368, 74

Roncarelli M., Villaescusa-Navarro F., Baldi M., 2017, MNRAS, 467, 985

Sakstein J., Jain B., 2017, Phys. Rev. Lett., 119, 251303

Schmidt B. P. et al., 1998, ApJ, 507, 46

Shaw L. D., Rudd D. H., Nagai D., 2012, ApJ, 756, 15

Sotiriou T. P., Faraoni V., 2010, Rev. Mod. Phys., 82, 451

Springel V., 2005, MNRAS, 364, 1105

Sunyaev R. A., Zeldovich Y. B., 1970, Ap\&SS, 7, 3

Trac H., Bode P., Ostriker J. P., 2011, ApJ, 727, 94

Ursino E., Galeazzi M., Roncarelli M., 2010, ApJ, 721, 46

Vainshtein A., 1972, Phys. Lett. B, 39, 393

Viel M., Lesgourgues J., Haehnelt M. G., Matarrese S., Riotto A., 2005, Phys. Rev. D, 71, 063534

Vikram V., Cabré A., Jain B., VanderPlas J. T., 2013, J. Cosmol. Astropart. Phys., 8, 020

Vishniac E. T., 1987, ApJ, 322, 597

Wetterich C., 1988, Nuclear Phys. B, 302, 668

Wetterich C., 1995, A\&A, 301, 321

Will C. M., 2006, Living Rev. Relativ., 9, 3

Winther H. A. et al., 2015, MNRAS, 454, 4208

Wright B. S., Winther H. A., Koyama K., 2017, J. Cosmol. Astropart. Phys., 10,054

Zel'dovich Y. B., 1970, A\&A, 5, 84

Zhao G.-B., 2014, ApJS, 211, 23

Zhao G.-B., Pogosian L., Silvestri A., Zylberberg J., 2009, Phys. Rev. D, 79,083513 